Check for updates

Cite this: J. Mater. Chem. C, 2017, 5,7270

\title{
Quantifying local thickness and composition in thin films of organic photovoltaic blends by Raman scattering $\dagger$
}

\author{
Xabier Rodríguez-Martínez, (D) *a Michelle S. Vezie, (D) ${ }^{\mathrm{b}}$ Xingyuan Shi, (D) ${ }^{\mathrm{b}}$ \\ lain McCulloch, (D) ${ }^{\text {cd }}$ Jenny Nelson, (D) ${ }^{b}$ Alejandro R. Goñi (iD ${ }^{\text {ae }}$ and \\ Mariano Campoy-Quiles (D)*a
}

\begin{abstract}
We report a methodology based on Raman spectroscopy that enables the non-invasive and fast quantitative determination of local thickness and composition in thin films (from a few monolayers to hundreds of $\mathrm{nm}$ ) of one or more components. We apply our methodology to blends of organic conjugated materials relevant in the field of organic photovoltaics. As a first step, we exploit the transfer-matrix formalism to describe the Raman process in thin films including reabsorption and interference effects of the incoming and scattered electric fields. This allows determining the effective solid-state Raman cross-section of each material by studying the dependence of the Raman intensity on film thickness. These effective cross sections are then used to estimate the local thickness and composition in a series of polymer:fullerene blends. We find that the model is accurate within $\pm 10 \mathrm{~nm}$ in thickness and \pm 5 vol\% in composition provided that (i) the film thickness is kept below the thickness corresponding to the first maximum of the calculated Raman intensity oscillation; (ii) the materials making up the blend show close enough effective Raman cross-sections; and (iii) the degree of order attained by the conjugated polymer in the blend is similar to that achieved when cast alone. Our methodology opens the possibility of making quantitative maps of composition and thickness over large areas (from microns to centimetres squared) with diffraction-limited resolution and in any multicomponent system based thin film technology.
\end{abstract}

Received 6th April 2017,
Accepted 21st June 2017

DOI: $10.1039 / \mathrm{c} 7 \mathrm{tc0} 01472 \mathrm{~d}$

rsc.li/materials-c

\section{Introduction}

Conjugated polymers and small molecules offer unique optical and semiconducting properties to build functional electronic devices with appealing features, including flexibility, lightness, versatility and low manufacturing cost. Organic photovoltaics

\footnotetext{
${ }^{a}$ Institut de Ciència de Materials de Barcelona (ICMAB-CSIC), Bellaterra, 08193, Spain.E-mail: xrodriguez@icmab.es, mcampoy@icmab.es

${ }^{b}$ Centre for Plastic Electronics and Department of Physics, Imperial College London, Prince Consort Road, London SW7 2AZ, UK

${ }^{c}$ Centre for Plastic Electronics and Department of Chemistry, Imperial College London, Exhibition Road, London SW7 2AZ, UK

${ }^{d}$ SPERC, King Abdullah University of Science and Technology, Thuwal 23955-6900, Saudi Arabia

${ }^{e}$ Institució Catalana de Recerca I Estudis Avançats (ICREA), Barcelona, 08010, Spain

$\dagger$ Electronic supplementary information (ESI) available: Further details about the application of the methodology in different Raman setups; the multi-wavelength fitting approach in P3HT films; the sensitivity of the methodology in thin film architectures with a high refractive index contrast; and the effect of photodegradation on the Raman intensity in some polymer:fullerene blends. See DOI: $10.1039 / \mathrm{c} 7 \mathrm{tc} 01472 \mathrm{~d}$
}

(OPV) is an example of an emerging technology that utilises the aforementioned properties in solar cells with good conversion efficiencies, currently exceeding $11 \% .^{1,2}$ The active layer in these devices typically consists of a blend of a hole transporting conjugated polymer and an electron transporting conjugated small molecule (usually a fullerene derivative), thoroughly mixed and cast from solution as a thin film forming the so-called bulk heterojunction (BHJ). ${ }^{3}$ The wet deposition process enables solid-state inhomogeneities to occur (e.g. phase segregation, thickness variations, etc.) that can extend up to different length scales. ${ }^{4}$ Solubility within the solvents, miscibility between components, surface energies and drying kinetics typically govern phase separation at the nanometer to micron scale. In turn, the size and purity of domains at that scale will have a strong impact on charge generation, recombination and transport in OPV blends. The characterization of film morphology at the nanoscale is well-documented through the use of atomic force microscopy (AFM), ${ }^{5}$ X-ray microscopy ${ }^{6}$ and more recently through energy-filtered scanning electron microscopy (EFSEM) ${ }^{7}$ as well as tip-enhanced optical spectroscopy (TEOS), including Raman and photoluminescence. ${ }^{8}$ Apart from nanometric qualitative 
images of the film surface and topography, scanning probe microscopies such as TEOS and AFM can provide quantitative chemical maps with a lateral resolution down to $20 \mathrm{~nm} .{ }^{8-11}$ In the case of EFSEM this value reduces below a nanometre. ${ }^{7}$

A less investigated solid state inhomogeneity is that occurring at the micron to centimetre length scales. This is the result of wet solution processing without full control of the rheology of the solution (mainly viscosity and surface tension), drying kinetics and substrate energies. Typical manifestations of this include poor wettability and adhesion, thickness oscillations, coffee-ring stains, and composition profiles due to Marangoni fluxes. All these prevent the formation of uniform and smooth films with well-defined interfaces, which in turn has detrimental effects on film reproducibility and technology upscalability. ${ }^{12,13}$ While these effects are often undesirable, it is worth noting that films exhibiting controlled lateral variations of thickness or composition (gradients) have been proposed in order to accelerate the screening of new OPV systems. ${ }^{14,15}$ In this case, variations in film thickness and/or composition extend over a scale of a few millimetres up to several centimetres.

The aforementioned high resolution techniques such as AFM or EFSEM could, in principle, be used to characterize large scale inhomogeneities. They would, however, be unaffordably slow. On the other hand, spectroscopic techniques such as ellipsometry may be exploited to speedily infer film thickness and composition in thin films at a larger scale, being applicable even in multi-layered stacks such as functional devices if the optical constants are known beforehand. ${ }^{16}$ The lateral resolution in ellipsometry is, however, limited by the size of the light spot used (typically greater than $200 \mu \mathrm{m}$ ), a fact that makes the study of the phase segregation on smaller length scales unfeasible. Moreover, the refractive index is only moderately selective to different materials.

Micro-Raman spectroscopy constitutes a scanning or imaging technique that can be exploited to study thin film morphology with diffraction-limited lateral resolution ( $c a .300 \mathrm{~nm}$ in the best scenario, i.e. using $488 \mathrm{~nm}$ as the excitation wavelength and an objective with a numerical aperture $\geq 0.9$ ), thus linking the two regimes explained above: nanoscale and macroscale characterization. Such resolution, in combination with the characteristic fingerprinting capability of Raman scattering, and rapid acquisition of data, enables the characterization of film inhomogeneities and phase segregation extending from a few microns up to centimetres in a single step. Raman spectroscopy also offers a series of unique advantages such as non-invasiveness and sensitivity to chemical environments. ${ }^{17-22}$ These features make Raman spectroscopy a valuable technique to gain insights regarding structure-performance relationships in organic thin films ${ }^{23}$ and to quantify the relative content of individual components according to their Raman fingerprint. ${ }^{24}$

Most studies regarding Raman measurements in $\pi$-conjugated thin films restrict themselves, with a few exceptions, ${ }^{21}$ to a qualitative analysis of the results. While Raman shifts and peak widths are useful to identify crystalline and amorphous domains, the analysis of the Raman intensity mainly reduces to the deconvolution of the acquired spectra as weighted linear combinations of the individual components ${ }^{25,26}$ and the relative identification of thicker or thinner regions based on a linear approximation of the measured Raman intensity. ${ }^{4}$ As we will show below, the validity of such approximations is rather limited.

Beyond organic semiconductors, Raman scattering is currently exploited to determine the thickness of supported films ${ }^{27}$ such as thin adsorbed layers, ${ }^{28,29}$ graphene ${ }^{30,31}$ and exfoliated chalcogenides ${ }^{32}$ according to the concept of interference-enhanced Raman scattering. ${ }^{33}$ The method is based on a detailed mathematical description of the incident and scattered electric field amplitudes in multi-layered structures, which interfere according to the optical properties of the whole stack. By properly tuning such properties as well as the thickness of the layers, the Raman intensity due to nanometric films can undergo up to a ten-fold increase compared to the bulk material. ${ }^{32}$

This paper extends these ideas of film thickness quantification to multi-component layers by enabling the simultaneous determination of film thickness and volumetric composition. Our methodology is based on rigorously modeling the distribution of the incoming electromagnetic field and the scattered light to account for the variations of the Raman intensity registered as a function of film thickness, in both pure materials and blends. In the latter, the raw spectra are fitted as a combination of the pure compounds using the thin film thickness and the volumetric scattering fraction as free parameters. The experimental results are in good agreement with those expected from the model and those extracted through ellipsometry provided that (i) the morphology and chemical micro-environment of the probed regions do not differ too much from those encountered for the thin film reference (pristine) materials; and (ii) the materials forming the blend show similar Raman cross-sections at the given excitation wavelengths. Moreover, the thin film thickness can be determined unequivocally if its value stays below the thickness corresponding to the first maximum of the Raman intensity oscillation predicted by the mathematical approach.

To the best of our knowledge this work constitutes the first report in which Raman scattering is used to quantify film thickness and volumetric scattering fractions (a.k.a. volumetric composition) in multi-component mixtures of conjugated materials deposited as thin films. This enables the imaging of thickness and composition with diffraction limited spatial resolution over areas from microns up to centimetres squared, thus bridging the two regimes currently addressed by other techniques (e.g. local probes for less than 25-by25 microns squared and ellipsometry/SIMS for averaged values over $\mathrm{cm}^{2}$ areas). Furthermore, the methodology itself is not restricted to a particular type of material or film architecture but it can be applied in any thin film technology which includes Raman-active chromophores: single- or multi-layered structures, free-standing or supported films, and organic or inorganic materials.

\section{Methodology}

\section{Mathematical framework}

The main scope of this work consists of developing and testing a suitable mathematical framework dictating the relationship 
between Raman intensity, film thickness and local volumetric composition in multi-component mixtures. We first consider that the Raman scattering process is divided into three steps: (1) the excitation of the film by the incident laser beam; (2) the generation of the Raman-scattered light from the film; and (3) the collection of the Raman light. The amplitude of the incident electromagnetic field determines the probability of Raman scattering to occur within the thin film, as we consider it proportional to the probability to induce oscillating dipoles. Such a term weights the intensity of the Raman-scattered light emitted from the bulk of the film, leading to interference effects in the dependence of the Raman intensity on film thickness curve (as we demonstrate in the Experimental section).

The distribution of an incoming electromagnetic field in a multi-layered system can be described following a broad variety of formalisms, the transfer matrix method being one of the most elegant approaches. ${ }^{34-36}$ In this case, each interface and layer is described by 2-by-2 matrices including their respective optical constants and thicknesses. Then, the electric field distribution in each layer can be calculated as a product of square matrices, assuming that the incident field is a timeindependent plane wave. This methodology is strictly valid if the layers are homogeneous and the interfaces are flat within the spot size of the incident beam.

Let us consider the case of a thin film (medium 1, with optical constants $n_{1}$ and $\kappa_{1}$ for the refractive index and extinction coefficient, respectively) deposited on top of a glass substrate (medium 2, semi-infinite) under vacuum (medium 0, semiinfinite). In a one-dimensional case and for normal incidence, the transfer matrix method provides a simple expression for the amplitude of the electric field at a film depth $x$ (from the vacuum interface) due to the incoming laser beam of wavelength $\lambda_{\mathrm{L}}$, which reads:

$$
E_{\mathrm{L}}(x)=\frac{t_{01}\left(\mathrm{e}^{\mathrm{i} \xi_{1} x}+r_{12} \mathrm{e}^{\mathrm{i} \xi_{1}(2 d-x)}\right)}{1-r_{10} r_{12} \mathrm{e}^{2 \mathrm{i} \xi_{1} d}}\left|E_{0}\right|=t_{\mathrm{L}}\left|E_{0}\right|
$$

where $\mathrm{i}$ is the imaginary unit, $t_{\mathrm{ab}}$ and $r_{\mathrm{ab}}$ are the complex Fresnel transmission and reflection coefficients of the interfaces between a and b media, $\xi_{1}=2 \pi\left(n_{1}+\mathrm{i} \kappa_{1}\right) / \lambda_{\mathrm{L}}, d$ is the film thickness and $\left|E_{0}\right|$ is the amplitude of the plane wave that describes the incoming field. For simplicity, $\left|E_{0}\right|$ is taken to unity. In practice, this is not a constraint as we normalize the Raman signal by the laser power arriving at the sample.

An analogous mathematical description applies also in the case of light emitted from the bulk of a film, a.k.a. the Ramanscattered light. We consider the probability of inducing oscillating dipoles in the media to be proportional to the incident electromagnetic field at the probed position of the thin film, which is then responsible for the Raman-scattered radiation according to the cross-section of the mode under study. Thus the amplitude of the scattered field $E_{\mathrm{R}}$ leaving the film towards the spectrometer can be written as

$$
E_{\mathrm{R}}(x) \propto \frac{t_{10}^{R}\left(\mathrm{e}^{\mathrm{i} \xi_{1}^{R} x}+r_{12}^{R} \mathrm{e}^{\mathrm{i} \xi_{1}^{R}(2 d-x)}\right)}{1-r_{10}^{R} r_{12}^{R} \mathrm{e}^{2 \mathrm{i} \xi_{1}^{R} d}}\left|E_{\mathrm{L}}(x)\right|=t_{\mathrm{R}}\left|E_{\mathrm{L}}(x)\right|
$$

where the superscript $R$ refers to the values attained at the Raman-scattered wavelength $\left(\lambda_{\mathrm{R}}\right)$. Since $E_{\mathrm{R}}(x)$ stands for the emergent Raman-scattered electric field amplitude due to an infinitesimal material slab located at a distance $x$ from the film surface, the total Raman intensity $I_{\mathrm{R}}$ is given by integrating the squared modulus of the scattered field $\left|E_{\mathrm{R}}(x)\right|^{2}$ (i.e. the scattered intensity due to such a slab) over the entire film thickness:

$$
I_{\mathrm{R}}(d)=\sigma_{\mathrm{R}} \int_{0}^{d}\left|E_{\mathrm{R}}(x)\right|^{2} \mathrm{~d} x
$$

where $\sigma_{\mathrm{R}}$ is an empirical, solid-state effective Raman crosssection that stands for the measured Raman activity (with units of counts $\mathrm{s}^{-1} \mathrm{~W}^{-1}$ ) of any mode under study. Eqn (3) results in an interference-like distribution for the Raman intensity as a function of film thickness. The oscillatory behaviour has the same origin as the oscillations of short circuit current as a function of film thickness observed in organic photovoltaics. ${ }^{35}$ Note that eqn (3) also takes into account the absorption (reabsorption) of the incoming (scattered) field as indicated by the complex refractive index included in the definition of $\xi_{1}\left(\xi_{1}^{R}\right)$. Clearly, this formalism can be extended for systems comprising a larger number of layers by calculating the corresponding Fresnel coefficients of the two subsets separated by the Raman-active layer, i.e. media 0 and 2.

Eqn (3) holds for incoming and scattered s- (TE) and p-polarized (TM) waves according to their complex Fresnel coefficients. ${ }^{35}$ This fact leads to slightly different Raman intensity distributions depending on the selected polarization combination and angle of incidence. Taking into account the polarization of the incident and scattered waves, eqn (3) can be rewritten as

$$
I_{\mathrm{R}}(d)=\sigma_{\mathrm{R}}\left|E_{0}\right|^{2} \int_{0}^{d}\left(\alpha\left|t_{\mathrm{L}}^{\mathrm{s}}(x)\right|^{2}+\beta\left|t_{\mathrm{L}}^{\mathrm{p}}(x)\right|^{2}\right) \cdot\left(\alpha\left|t_{\mathrm{R}}^{\mathrm{s}}(x)\right|^{2}+\beta\left|t_{\mathrm{R}}^{\mathrm{p}}(x)\right|^{2}\right) \mathrm{d} x
$$

where $\alpha$ and $\beta$ are the weighting factors associated with s- and p-polarizations such that $\alpha+\beta=1$. Interestingly, we found that despite performing backscattered Raman experiments (i.e. in normal incidence) the intensity values oscillate differently with film thickness depending on the numerical aperture of the objective as well as on the type of sample geometry being measured. Microscope objectives with low magnification and numerical aperture $(10 \times, \mathrm{NA}=0.25)$ lead to data fits in which $\alpha \approx 1$, indicating that the incident electric field is mostly in normal incidence within the dimensions of the laser spot. Furthermore, $\alpha \approx 1$ when collecting the Raman-scattered light through a thick superstrate such as a glass slide $(n=1.45$, $1.2 \mathrm{~mm}$-thick) even when using objectives with larger magnification and numerical aperture $(40 \times, \mathrm{NA}=0.6)$. In this case, the refractive index contrast between air $(n=1)$ and glass $(n=1.45)$ reduces the refraction angle of the rays impinging obliquely, thus enhancing the weight of the s-component in eqn (4). For moderate magnifications and numerical apertures, and in the absence of thick incoherent superstrates, eqn (4) generally leads to fits in which $\alpha=\beta=0.5$, thus equally mixing all incident and scattered polarizations. 
Regarding multi-component mixtures, the intensity of the acquired Raman spectra can be modeled in order to locally estimate both film thickness $(d)$ and volumetric composition $\left(v_{u}\right)$ in medium 1 . To do so we assume that in a thin film of $N$ different components the measured Raman signal corresponds to a superposition of the spectra obtained for the pure compounds forming the blend. Hence the Raman intensity at any location in the sample can be described quantitatively as a linear combination of the basis (reference) spectra for the pure compounds. Thus, accurate quantitative information can be obtained if the effective cross-sections of the individual components are known. Generalizing for a blend of $N$ components the Raman intensity reads

$$
I_{\mathrm{R}, \operatorname{mix}}\left(d, v_{u}, \bar{v}\right) \propto \int_{0}^{d}\left|E_{\mathrm{R}}\left(x, v_{u}\right)\right|^{2} \mathrm{~d} x \sum_{u=1}^{N} v_{u} \sigma_{\mathrm{R}, u} I_{\mathrm{ref}, u}(\bar{v})
$$

where $\bar{v}$ corresponds to the Raman shift; $I_{\text {ref }, u}(\bar{v})$ stands for the (reference) Raman spectrum of component $u$ normalized the intensity of the mode associated with the cross-section $\sigma_{\mathrm{R}, u} ; v_{u}$ is the volumetric scattering fraction of component $u$ (which satisfies $\sum_{u=1}^{N} v_{u}=1$ ), and $d$ refers now to the thickness of the blended thin film in the probed region. For the Ramanscattered radiation $E_{\mathrm{R}}\left(x, v_{u}\right)$ the optical constants are assumed to vary linearly with the volumetric composition as $\varepsilon_{\operatorname{mix}}=\sum_{u=1}^{N} v_{u} \varepsilon_{u}$, so we implicitly assume that they are well mixed at the $\lambda_{\mathrm{L}}$-length scale. We have also tested other effective medium approximations for $\varepsilon_{\text {mix }}$ such as Bruggeman's model and obtained results for the fit which lay within the statistical error of the linear approximation proposed (for the blends measured in this work); consequently, we adopted the latter to keep the methodology as simple as possible.

The rigorous description of the Raman-scattered radiation with the transfer matrix method presented here is compared with three simpler approaches. First, a thickness-independent approximation for the scattered light,

$$
I_{\text {mix }, \text { ind }}\left(v_{u}, \bar{v}\right)=\sum_{N} v_{u} \sigma_{\mathrm{R}, u} I_{\mathrm{ref}, u}(\bar{v})
$$

This is a typical approximation used for bulk multi-component materials. Second, a linear dependence of the Raman intensity with film thickness,

$$
I_{\text {mix }, \operatorname{lin}}\left(d, v_{u}, \bar{v}\right)=d \sum_{N} v_{u} \sigma_{\mathrm{R}, u} I_{\mathrm{ref}, u}(\bar{v})
$$

This has been previously used to model blend films. ${ }^{4}$ Finally, an exponential decay of the incoming and scattered fields within the film,

$$
I_{\text {mix }, \text { ex }}\left(d, v_{u}, \bar{v}\right)=\int_{0}^{d} \mathrm{e}^{-\left(\alpha\left(v_{u}\right)+\alpha_{\mathrm{R}}\left(v_{u}\right)\right) x} \mathrm{~d} x \sum_{N} v_{u} \sigma_{\mathrm{R}, u} I_{\mathrm{ref}, u}(\bar{v})
$$

where $\alpha\left(v_{u}\right)=4 \pi \kappa_{1}\left(v_{u}\right) / \lambda_{\mathrm{L}}$ and $\alpha_{\mathrm{R}}\left(v_{u}\right)=4 \pi \kappa_{1}^{R}\left(v_{u}\right) / \lambda_{\mathrm{R}}$. This would represent the simplest (Beer-Lambert-like) model that can account for two important experimental facts, the initial increase in the Raman signal with the amount of material (thickness) and the saturation of this behaviour for thick films (much greater than the penetration depth of the light).

\section{Experimental validation and protocol}

We experimentally validate the methodology using the following protocol:

(1) First, a thickness series of uniform, homogeneous samples containing a single, pure material is prepared. An alternative that we have extensively explored here is to use samples with a thickness gradient over relatively large lateral distances so as to be uniform within the laser spot. By scanning the laser beam across the sample, many experimental points can be obtained.

(2) Then, the Raman intensity (effective counts per second) of a particular band (usually the most intense one) is tracked as a function of film thickness.

(3) The experimental data are fitted according to eqn (4) using $\sigma_{\mathrm{R}}$ as a free parameter after measuring the film thickness through profilometry, AFM or ellipsometry at the same locations where Raman spectra were acquired. Thus $\sigma_{\mathrm{R}}$ is a material property associated with a given mode and excitation wavelength. Proper background subtraction is necessary to avoid photoluminescence disrupting the observed trends. Note that for the calculation of eqn (4) we need the optical constants of the whole multi-layered stack as input parameters (see Table 1). They can often be taken from the literature, ${ }^{16}$ or measured by complementary techniques such as ellipsometry.

The previous steps (1-3) are only required to experimentally verify that the oscillations of the Raman intensity with film thickness are well described by the mathematical model. When implementing the methodology to measure the Raman signal in some of the materials provided in Table 1, a few samples of the material are sufficient to measure the Raman spectrum and thickness locally. Then, by fitting the corresponding $I_{\mathrm{R}}(d)$ curve, the effective Raman cross-section for that material in that particular Raman setup is extracted; the term effective refers to the fact that such a value is only valid for that setup, including optics (objective magnification and numerical aperture) and CCD yield. Finally, by using the ratiometric values provided in Table 1 the method can be implemented readily in pure or mixed films of any materials for which $\sigma_{\mathrm{R}}$ has been determined. These ratios remain constant regardless of the Raman setup and optics used (Fig. S1 in the ESI $\dagger$ ). Thus once $\sigma_{\mathrm{R}}$ is determined for a given material (and keeping the geometrical measuring factors constant, including the numerical aperture and objective magnification), this value can be used in combination with eqn (4) to obtain an unknown thickness for pristine films on any substrate, to evaluate film homogeneity, or to monitor film thickness in-line. When extending the methodology to materials beyond those listed in Table 1 , steps 1 to 3 are advised to get proper statistics and error estimations on effective $\sigma_{\mathrm{R}}$ values for a particular Raman setup.

When evaluating multi-component systems, the following steps need to be taken:

(4) In multi-component mixtures we first normalize every material spectrum by the intensity of the Raman mode associated with the previously estimated cross-section, $\sigma_{\mathrm{R}}$, as well as by the laser power impinging at the sample. The normalization step 
Table 1 Collection of ratiometric Raman cross-sections (with respect to $\mathrm{PC} \mathrm{C}_{70} \mathrm{BM}$ ) for the conjugated materials analysed in this work. The uncertainties

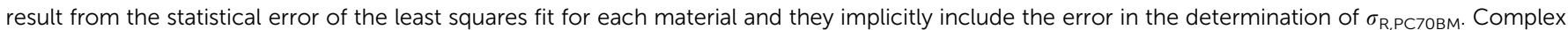
refractive indices at both the excitation wavelength and at the Raman-scattered wavelength associated with the corresponding vibrational band are also included. The film thickness corresponding to the first Raman intensity maximum serves as a reference to evaluate the single valued fitting regime of the methodology

\begin{tabular}{|c|c|c|c|c|c|c|c|c|c|}
\hline \multirow[b]{2}{*}{ Material } & \multicolumn{2}{|l|}{$\sigma_{\mathrm{R}, u} / \sigma_{\mathrm{R}, \mathrm{PC} 70 \mathrm{BM}}$} & \multicolumn{2}{|c|}{$\begin{array}{l}\text { Complex refractive } \\
\text { index at } \lambda_{\mathrm{L}}\end{array}$} & \multirow{2}{*}{$\begin{array}{l}\text { Raman band } \\
\left(\lambda_{\mathrm{R}}\right) \mathrm{cm}^{-1}\end{array}$} & \multicolumn{2}{|c|}{$\begin{array}{l}\text { Complex refractive } \\
\text { index at } \lambda_{\mathrm{R}}\end{array}$} & \multicolumn{2}{|c|}{$\begin{array}{l}\text { Thickness of first } \\
\text { Raman intensity maximum }\end{array}$} \\
\hline & $514 \mathrm{~nm}$ & $488 \mathrm{~nm}$ & $514 \mathrm{~nm}$ & $488 \mathrm{~nm}$ & & $514 \mathrm{~nm}$ & $488 \mathrm{~nm}$ & $514 \mathrm{~nm}$ & $488 \mathrm{~nm}$ \\
\hline C16-IDTBT $^{a}$ & $5.2 \pm 0.7$ & $8 \pm 1$ & $1.35+0.31 \mathrm{i}$ & $1.42+0.18 \mathrm{i}$ & 1353 & $1.41+0.51 \mathrm{i}$ & $1.35+0.36 \mathrm{i}$ & $162 \mathrm{~nm}$ & $500 \mathrm{~nm}$ \\
\hline DPP-TT-T- ${ }^{b}$ & $1.3 \pm 0.2$ & $4.3 \pm 0.5$ & $1.41+0.13 \mathrm{i}$ & $1.48+0.17 \mathrm{i}$ & 1502 & $1.16+0.22 \mathrm{i}$ & $1.35+0.08 \mathrm{i}$ & $>500 \mathrm{~nm}$ & $>500 \mathrm{~nm}$ \\
\hline DPP-TT-T- $3^{b}$ & $1.4 \pm 0.2$ & $4.4 \pm 0.5$ & $1.11+0.27 i$ & $1.25+0.24 \mathrm{i}$ & 1502 & $0.97+0.52 \mathrm{i}$ & $1.06+0.33 \mathrm{i}$ & $151 \mathrm{~nm}$ & $>500 \mathrm{~nm}$ \\
\hline $\operatorname{PCBM}^{a}$ & $0.15 \pm 0.04$ & $0.3 \pm 0.1$ & $2.02+0.09 \mathrm{i}$ & $2.04+0.11 \mathrm{i}$ & 1461 & $2.01+0.07 i$ & $2.02+0.09 \mathrm{i}$ & $145 \mathrm{~nm}$ & $135 \mathrm{~nm}$ \\
\hline rr-P3HT ${ }^{a}$ & $51 \pm 5$ & $59 \pm 6$ & $1.74+0.95 i$ & $1.50+0.88 i$ & 1450 & $2.15+0.85 i$ & $1.84+0.91 \mathrm{i}$ & $103 \mathrm{~nm}$ & $109 \mathrm{~nm}$ \\
\hline $\mathrm{PC}_{70} \mathrm{BM}^{a}$ & 1 & 1 & $2.14+0.35 i$ & $2.08+0.40 \mathrm{i}$ & 1564 & $2.19+0.29 \mathrm{i}$ & $2.15+0.33 i$ & $122 \mathrm{~nm}$ & $116 \mathrm{~nm}$ \\
\hline
\end{tabular}

implies that within the spectral region studied (usually within the range $1000-1700 \mathrm{~cm}^{-1}$ ) the spectrum varies uniformly with film thickness and laser power as dictated by the intensity oscillation of the band associated with $\sigma_{\mathrm{R}}$. This approximation is valid provided that relative Raman band intensities are constant upon variations of film thickness and/or composition. We repeat this procedure for all the materials forming the mixture to build a library of effective Raman cross-sections for each material $\left(\sigma_{\mathrm{R}, u}\right)$ in our particular setup as well as their corresponding normalized spectra $\left(I_{\text {ref }, u}(\bar{v})\right)$.

Finally, we use eqn (5) to fit the raw Raman spectra of the multi-component mixtures using film thickness $d$ and volumetric fraction $v_{u}$ as free parameters. Once again, a correct background subtraction is required for proper data fitting. Note that backgrounds may be very different in pristine and blend films, since photoluminescence is often quenched by mixing.

\section{Results and discussion}

In a first set of experiments, we evaluated the Raman spectra of three soluble fullerenes and eleven different conjugated polymers; see Fig. 1. Their Raman spectra are generally featurerich and characteristic enough so as to clearly identify their vibrational fingerprints in multi-component films. This is important as blends of conjugated polymers and fullerenes are the main material systems used as the active layer in organic solar cells. The spectral window between $1000 \mathrm{~cm}^{-1}$ and $1700 \mathrm{~cm}^{-1}$, corresponding to modes associated with carbon single and double bonds, exhibits peaks that are both generally strong for all materials and centered at material-specific positions.

In a second series of experiments, we determined the single material effective solid-state Raman cross-sections, $\sigma_{\mathrm{R}}$, for a selection of materials. For this purpose, we used films exhibiting a lateral thickness gradient (see the Experimental section) as they enable the local measurement of the Raman spectra in order to then correlate the intensity with the thickness profile obtained through profilometry, ellipsometry or AFM. A typical thickness gradient spanned between $20 \mathrm{~nm}$ and $150 \mathrm{~nm}$ across over $5 \mathrm{~cm}$ in sample length. This means that over the spot size of our laser (around 20 microns) the thickness varies less than $0.06 \mathrm{~nm}$ and thus the sample can be considered locally homogeneous. Interestingly, with this approach we are able to combine in a single sample the equivalent information that we would have obtained by fabricating several tens of them, saving both material and time.

Fig. 2(a-d) illustrate the variation of the Raman intensity with film thickness for four conjugated materials under different excitation wavelengths: (a) $\mathrm{PC}_{70} \mathrm{BM}$ and (b) PCPDTBT at $514 \mathrm{~nm}$ excitation, and (c) C16-IDTBT and (d) PCDTBT at $488 \mathrm{~nm}$ excitation. Additional data for these materials and P3HT can be found in Fig. S2 and S3 in the ESI. $\dagger$ Three of these compounds are often used as active materials in OPVs. The fourth, C16-IDTBT, is a low band gap polymer that shows a high field-effect mobility as well as a relatively insensitive polymer structure when the processing conditions are varied. ${ }^{37,38}$ Branched side-chain analogues have been used to fabricate efficient solar cells. ${ }^{39,40}$ The experimental data were extracted from a single graded sample supported on glass, whose thickness as a function of position was double-checked using profilometry. Marks were performed at the beginning and at the end of the measuring region, and Raman spectra were acquired at spatial intervals of $500 \mu \mathrm{m}$. Note that profilometric line scans are not acquired exactly at the same position where Raman was measured, but with an uncertainty of a few tenths of microns. Thus if the sample presents thickness inhomogeneities which extend over that scale, then the correlation between Raman intensity and film thickness may deviate slightly. This effect is also present if ellipsometry is used to measure the film thickness due to the difference in the diameter of the spot used in both techniques ( $c a .20 \mu \mathrm{m}$ in our Raman setup $v s .>200 \mu \mathrm{m}$ in ellipsometry). Homogeneous samples were prepared using both blade and spin coating to verify that the casting method does not have a noticeable influence on the Raman intensity of the modes under study, or the graded profile of the sample.

The general trend is that the Raman intensity slowly increases with thickness until it reaches an asymptotic maximum. 


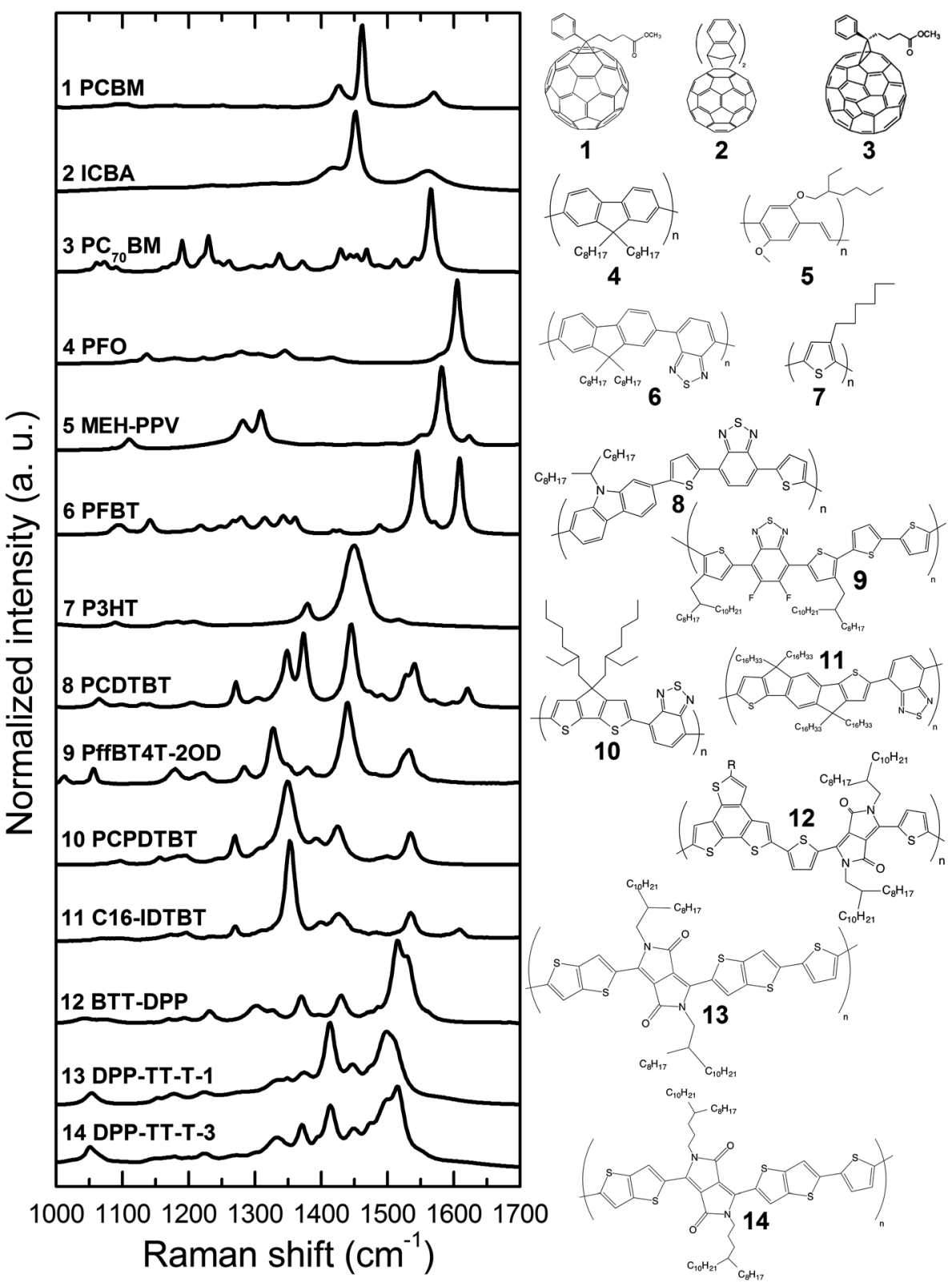

Fig. 1 Normalized Raman spectra of the conjugated polymers and fullerenes measured in this work including their chemical structure.

Additionally, there are mild oscillations of the intensity prior to reaching such an asymptote, whose amplitude and period depend on the optical constants of the whole layered stack at the excitation and scattered wavelengths. The solid lines in Fig. 2 represent a non-linear, least squares fit of the interference curve obtained by integrating eqn (4) for each material and wavelength taking $\alpha=\beta=0.5$. For comparison, the thickness-independent (dashed-dotted line, eqn (6)), the linear (dotted line, eqn (7)) and the exponential (dashed line, eqn (8)) models are also plotted and fitted by least-squares to the experimental data (except the thickness-independent model, which matches the asymptote of the exponential model). While both the thickness-independent and the linear approximations are clearly too simple to explain the experimental trends, both the exponential and transfer matrix models fit the raw data reasonably well in most cases.
The exponential approximation, despite being such a simple approach, well reproduces the trend in most conjugated polymers given the absence of strong interference effects. Nevertheless, these are progressively more critical as the refractive index contrast between adjacent films increases, in which case the transfer matrix model provides the correct trends; see Fig. S4 in the ESI $\dagger$ for a detailed study of C16-IDTBT Raman mode intensities when deposited on a silicon substrate. Thus, as the complexity of the multi-layered stack increases, the accuracy of the transfer-matrixbased model is significantly enhanced compared to other simpler approximations. To demonstrate this fact we measured a PCDTBT sample showing a lateral thickness gradient in which part of its exposed surface was covered with a thermally evaporated $\mathrm{MoO}_{3} / \mathrm{Ag}$ electrode, whose thicknesses are $12 \mathrm{~nm}$ and $100 \mathrm{~nm}$, respectively. Since the film was supported on a glass substrate $(1.2 \mathrm{~mm}$ thick) 


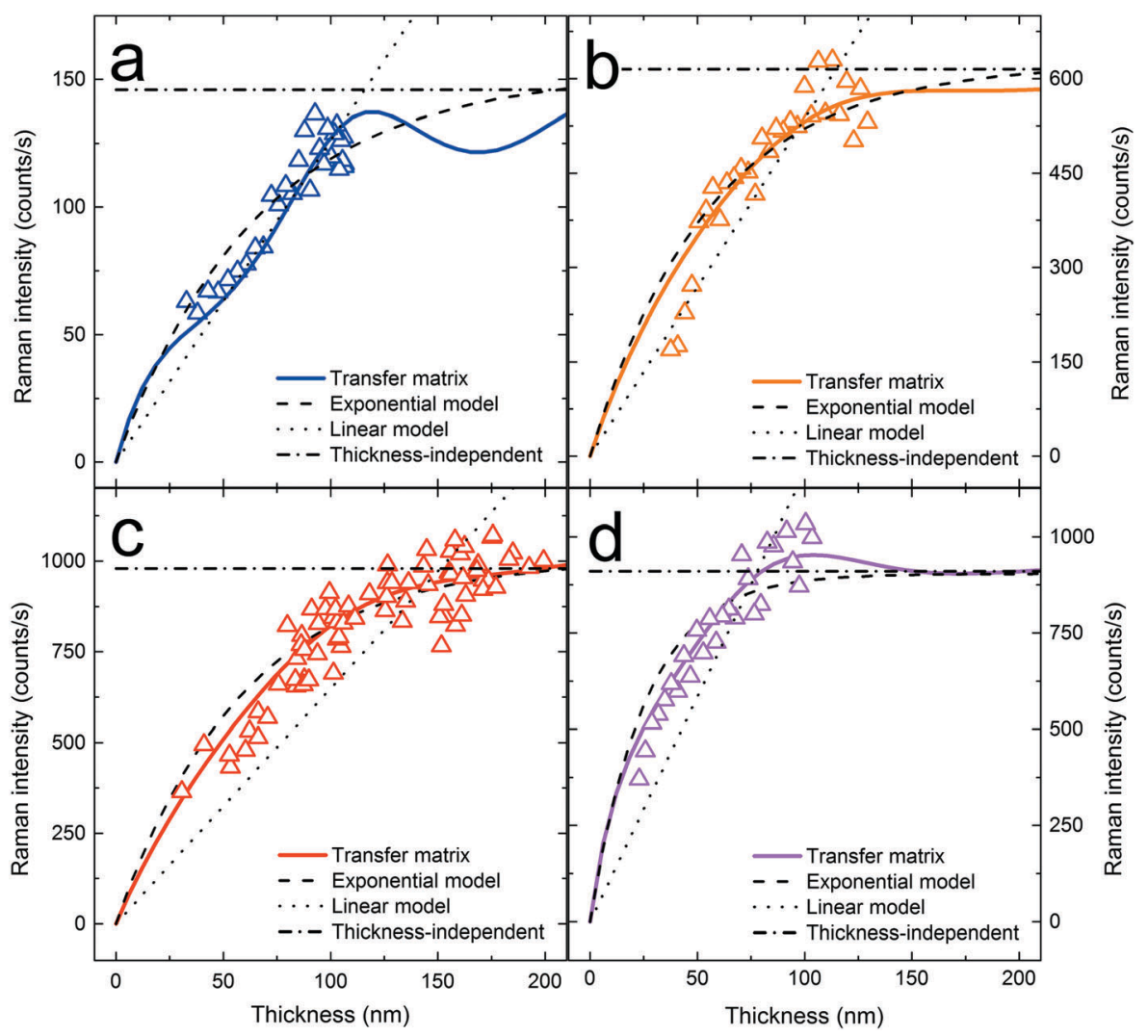

Fig. 2 Raman intensity (triangles) as a function of film thickness for (a) PC 70 BM under $514 \mathrm{~nm}$ excitation (1564 $\mathrm{cm}^{-1}$ band); (b) PCPDTBT under $514 \mathrm{~nm}$ excitation (1349 $\mathrm{cm}^{-1}$ band); (c) C16-IDTBT under $488 \mathrm{~nm}$ excitation (1353 $\mathrm{cm}^{-1}$ band); and (d) PCDTBT under $488 \mathrm{~nm}$ excitation (1445 cm ${ }^{-1}$ band). Solid lines stand for the transfer matrix modeling of the Raman process. Thickness-independent (dashed-dotted lines), linear (dotted lines) and exponential (dashed lines) models are also plotted and fitted to the experimental data to ease the comparison between approaches.

we focused the incident laser through such a thick incoherent layer, adding more complexity to the modeling of the electric field amplitudes. This structure resembles that of devices, in which the optical access is through the substrate. Fig. 3 illustrates the Raman intensity oscillations that occur in both the exposed and the covered regions using a $10 \times$ objective to focus through the glass, taking $\alpha=1$ and assuming that the (semi-infinite) incident medium is the glass substrate $\left(n_{0}=1.45\right)$. According to Fig. 3 , the three-fold intensity enhancement driven by the presence of the $\mathrm{MoO}_{3} / \mathrm{Ag}$ electrode is reproduced and the experimental data are properly fitted using the transfer matrix model in such a complex stack. Moreover, the single free-parameter used to fit the model to the experimental data, i.e. the effective Raman cross-section of PCDTBT $\left(\sigma_{\mathrm{R}, \mathrm{PCDTBT}}\right)$, acquires equivalent values regardless of the configuration of the multi-layered stack in which the polymer film is embedded. According to Fig. 3, the effective Raman cross-section of PCDTBT at $488 \mathrm{~nm}$ excitation reads $(2.1 \pm 0.2)$ counts s ${ }^{-1} \mathrm{~mW}^{-1}$ and $(2.1 \pm 0.1)$ counts $\mathrm{s}^{-1} \mathrm{~mW}^{-1}$ when such a polymer film is measured through the glass (glass/polymer/air) and with $\mathrm{a} \mathrm{MoO}_{3} / \mathrm{Ag}$ electrode (glass/polymer $/ \mathrm{MoO}_{3} / \mathrm{Ag}$ ), respectively. This experiment confirms the validity of our approach to model the Ramanscattered intensity even in model device systems as well as the generality of the effective Raman cross-sections extracted therein.

The relatively good match of the experimental data with the theoretical curves demonstrates that Raman spectroscopy can

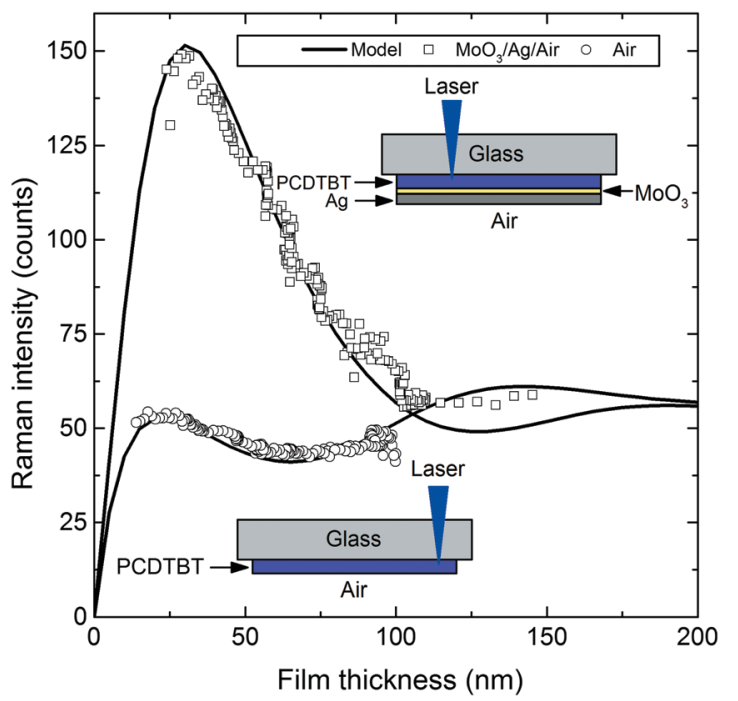

Fig. 3 Raman intensity as a function of thickness for a PCDTBT film (1445 $\mathrm{cm}^{-1}$ mode) deposited on a thick glass substrate $(1.2 \mathrm{~mm})$ in two different configurations: with (upper curve) and without (lower curve) a thermally evaporated $\mathrm{MoO}_{3} / \mathrm{Ag}$ electrode. Data took under ambient conditions using $488 \mathrm{~nm}$ excitation and a $10 \times$ magnification (NA $=0.25$ ) microscope objective to focus through the thick glass layer ( $\alpha=1$ in the modeling). The experimental data are normalized to the laser power used to measure the region without the $\mathrm{MoO}_{3} / \mathrm{Ag}$ electrode. 
also be used to rapidly determine the thickness in neat thin films, regardless of the deposition technique employed to prepare them. The accuracy of such determination is $c a$. $10 \mathrm{~nm}$, which renders the method as reliable as profilometry when dealing with soft films. This accuracy corresponds to the variance in the adjustable parameters as obtained from the least squares fit. Nevertheless, a qualitative inspection of the theoretical curve shows that its oscillatory behaviour constrains the accuracy of the methodology regarding film thickness determination. As a rule of thumb, if the thin film thickness is close to the thickness of the first Raman intensity maximum (Table 1), the Raman intensity vs. film thickness dependence is no longer single-valued due to the oscillation of the intensity and the accuracy is lowered up to $c a .20 \mathrm{~nm}$. Then (a) alternative measurement techniques should be performed to aid the estimation of the correct thickness; or (b) a different fitting routine should be implemented. In the latter case, we propose following a spectroscopic, multi-wavelength Raman approach in which the Raman spectra obtained at several wavelengths are fitted simultaneously with shared parameters (thickness and also composition in multi-component films). The result is then valid as long as the fitted thickness stays below the intensity saturation regime of the corresponding $I_{\mathrm{R}}(d)$ curve, which occurs at different values depending on the optical constants of the material at the excitation wavelength. P3HT constitutes an example in which such an approach can be applied successfully; see Fig. S3 in the ESI. $\dagger$

An important feature of the methodology presented in this work is that the values of the effective Raman cross-sections extracted from fitting eqn (4) to experimental data mainly depend on the geometry and optics of each Raman setup; in other words, their relative values are in principle constant if the same geometry (backscattering), optics and acquisition parameters at the CCD are used. This fact allows us to create a library of effective solid-state Raman cross-sections for each compound and excitation wavelength in the backscattering configuration (Table 1). In principle, if a material included in the library can be measured in a different Raman setup, Table 1 provides the general ratiometric values for the effective crosssections of the rest of the materials listed, thus enabling the facile implementation of the methodology in a different lab. Reassuringly we have been able to verify this by extracting the effective Raman cross-sections of several materials in two different confocal Raman setups and in a variety of substrates and layered structures while using objectives with different magnifications and numerical apertures; see Fig. S1 in the ESI. $\dagger$ The reader is referred to steps 1 to 3 of the experimental protocol for the estimation of cross-sections for materials other than those listed in Table 1.

So far we have discussed the modeling of the Raman signal for thin films of a single component. We next discuss the application of the methodology for blends of two components. In the case of well mixed multi-component blends, we show in the Appendix that the Raman interference curve also changes as a function of composition, with the maxima varying smoothly from the thickness value that would correspond to one component, to that of the other, following a behaviour governed by the assumed linear variation of the optical constants (see Fig. 6). There are two important requirements for the Raman signal of each component that makes the fitting of the corresponding mixture accurate. First, the Raman fingerprints of its components should be clearly distinguished through a wide range of compositions, which, according to Fig. 1, is the case for most material combinations; and secondly, the difference between Raman cross-sections cannot be too large (Table 1). For the latter our experience indicates that the ratio between Raman cross-sections should not be higher than 30 to cover the entire composition diagram (from $c a .5 \mathrm{vol} \%$ to $c a .95 \mathrm{vol} \%$ ) with acceptable accuracy. In some cases this can be achieved by properly selecting the excitation wavelength so that one of the components enters in resonance while the other is not. This is applicable to materials that exhibit complementary absorption spectra over some range of the spectrum.

In the next series of experiments, the methodology for multicomponent systems was tested by analysing two types of stateof-the-art polymer:fullerene blends used for OPVs. We selected two conjugated polymers, namely PCPDTBT (partially crystalline) and PCDTBT (rather insensitive to processing), and blended them with the fullerene $\mathrm{PC}_{70} \mathrm{BM}$ in variable weight ratios. By choosing these combinations we expected to cover two rather different cases of blending as far as morphology is concerned: while in the former case the semi-crystallinity of the polymer is likely to be partially disturbed by the presence of the fullerene, ${ }^{41}$ in the latter case the morphology of the polymer is expected to be less sensitive to fullerene loading. ${ }^{42-44}$ Both systems fulfil the a priori requirements mentioned above: they show close enough Raman cross-sections (Table 1) and clearly distinguishable Raman spectra in the region of interest (Fig. 1, $1000-1700 \mathrm{~cm}^{-1}$ ). The accuracy of Raman scattering in determining volumetric composition is assessed by comparing the values obtained with our methodology with results from variable-angle spectroscopic ellipsometry (VASE) in the same samples and locations. For the fit of the ellipsometric data, we assumed a Bruggeman's effective medium approximation and no vertical segregation through the film. During the modeling of the Raman intensity we double-checked that the use of a linear combination rather than a Bruggeman's effective medium approximation for the optical constants of the blended film introduced an uncertainty which was within the statistical error of the subsequent fit of the spectra.

Fig. 4(a and b) illustrate the volumetric compositions extracted through both techniques (Raman and VASE) for a series of PCDTBT:PC ${ }_{70} \mathrm{BM}$ and PCPDTBT:PC ${ }_{70} \mathrm{BM}$ blends deposited as thin films. Since the Raman-based values are plotted as a function of the ellipsometric composition, a straight line of slope unity would represent perfect matching between techniques. According to Fig. 4(a and b), Raman spectroscopy matches ellipsometry when determining the composition in PCDTBT: $\mathrm{PC}_{70} \mathrm{BM}$ blends whereas the techniques disagree in PCPDTBT: $\mathrm{PC}_{70} \mathrm{BM}$ films. Fig. 4(a and b) also indicate a small mismatch between the Raman-estimated polymer fractions depending on the excitation wavelength employed, with the estimated average 
polymer loading being generally higher under $514 \mathrm{~nm}$ excitation than $488 \mathrm{~nm}$.

The accuracy of the methodology in determining the film composition mainly depends on how distinguishable the Raman fingerprint spectra are as well as on the cross-section ratio of the blended materials. The optimum case would correspond to that in which the cross-section ratio is as close to unity as possible; under these circumstances, the accuracy of the Raman methodology is comparable to that of ellipsometric measurements ( $\pm 5 \mathrm{vol} \%)$ at moderate compositions (20-80 vol\%) but is lower in the extreme cases (below 10 vol\%). The former situation closely corresponds to the case of PCPDTBT:PC ${ }_{70} \mathrm{BM}$ blends (with a cross-section ratio of ca. 4), in which the statistical error bars due to the fit are of the order of $\pm 5 \%$ and are lower as the content of $\mathrm{PC}_{70} \mathrm{BM}$ increases (which has the lowest cross-section of both). On the other hand, the large cross-section ratio between PCDTBT and $\mathrm{PC}_{70} \mathrm{BM}$ (ca. 20) lowers the accuracy in the determination of the composition as the film gets polymer-enriched. The origin of such larger uncertainty is related to the fact that as the polymer content

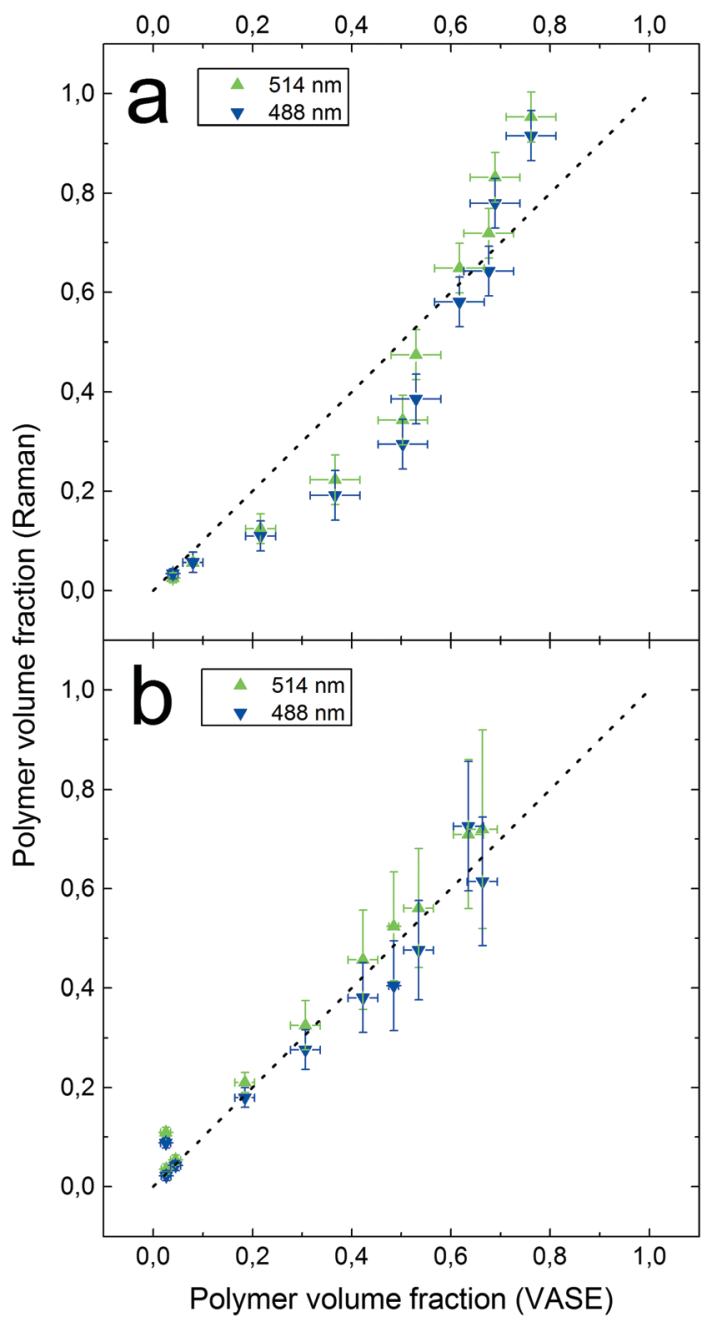

Fig. 4 Polymer volumetric fractions in (a) PCPDTBT:PC ${ }_{70} B M$ and (b) PCDTBT: $\mathrm{PC}_{70} \mathrm{BM}$ blended films as extracted through Raman ( $Y$ axis) and VASE ( $X$ axis). A straight dotted line is added as a guide-to-the-eye representing perfect matching between techniques. in the film increases the Raman fingerprint due to the fullerene is progressively hindered and the fit starts losing sensitivity to the presence of fullerene. Nevertheless, even in this adverse case the accuracy of the methodology is close to $\pm 10 \%$ in volume fraction (statistically).

Regarding the disagreement between Raman spectroscopy and ellipsometry in PCPDTBT:PC ${ }_{70} \mathrm{BM}$ blends, we ascribe it to the structural and conformational changes that take place upon blending, with the disruption to polymer crystallinity dependent on the fullerene loading. ${ }^{24,41,45}$ Since we have estimated the solid-state Raman cross-section of PCPDTBT using a pure graded film, the trend observed for the Raman intensity may not extrapolate perfectly well to the case of a blended film if its morphology or local density has changed significantly. At the same time, we expect variations of the optical constants to occur $^{23}$ which can explain the mismatch in the composition estimated at different excitation wavelengths. The optical constants are affected by the fullerene loading, the casting conditions (see Fig. S5 in the ESI $\dagger$ ) and the annealing post-treatments, which modify the aggregation and crystallinity of both components. ${ }^{45}$ This typically results in blue-shifts of the absorption and a reduction in anisotropy. It has been reported that upon blending with fullerenes, conjugated polymer chains may attain a less anisotropic orientation which eventually reduces their extinction coefficient up to one third of the value observed for pure films. ${ }^{46}$ Moreover, since the ellipsometric data are fitted according to a Bruggeman's model using as input parameters the optical constants for the pure PCPDTBT and $\mathrm{PC}_{70} \mathrm{BM}$ films, slight deviations of the volumetric composition and thickness are also expected following this approach. As we show in Fig. S4 and S5 in the ESI, $\dagger$ variations in the optical constants can have significant effects on the resulting Raman intensity dependence on film thickness. Thus, we expect Raman spectroscopy and ellipsometry to differ in those cases in which the morphology and the optical properties of the film are noticeably affected by the blending process. Conversely, the morphology in PCDTBT:PC ${ }_{70} \mathrm{BM}$ blends was reported to be highly robust, with the polymer phase within the blend being as amorphous as in the neat films and $\mathrm{PC}_{70} \mathrm{BM}$ domains aggregating with a low degree of long-range order. ${ }^{47}$ We have not observed any peak shifting upon blending nor variations in the relative peak intensities at any excitation wavelength even under ambient conditions, which constitutes a usual signature of robust morphology. Hence we expect the local densities and cross-sections to correlate well with those extracted for the pure films, explaining the improved matching of Raman and ellipsometry in the characterization of these samples.

We further explore the generality of the method by testing other binary blends (Table 2), including novel low band-gap co-polymers with promising power conversion efficiencies in organic solar cells, such as DPP-TT-T and BTT-DPP. ${ }^{48-50}$ According to Table 2, DPP-TT-T blends show consistent volumetric composition and lower film thickness values between VASE and Raman, whereas both techniques disagree in BTT-DPP blends. These results suggest that some of the above-mentioned blending effects that occur in PCPDTBT: $\mathrm{PC}_{70} \mathrm{BM}$ films are also taking place in BTT-DPP blends, which requires a more detailed analysis of 
Table 2 Comparison of polymer volumetric composition and thin film thickness as obtained from Raman spectroscopy and extracted through VASE in a series of polymer:fullerene blends. Raman values were fitted averaging at least three spectra at random positions in the sample

\begin{tabular}{|c|c|c|c|c|c|c|}
\hline Blend (w:w) & Vol\% (VASE) & \multicolumn{2}{|c|}{ Vol\% (Raman) } & Thickness (VASE) & \multicolumn{2}{|c|}{ Thickness (Raman) } \\
\hline DPP-TT-T-1: PC $_{70}$ BM $(1: 2)$ & $30 \pm 5 \%$ & $32 \pm 4 \%$ & $27 \pm 4 \%$ & $71 \pm 5 \mathrm{~nm}$ & $34 \pm 5 \mathrm{~nm}$ & $45 \pm 5 \mathrm{~nm}$ \\
\hline BTT-DPP : PC $_{70} \mathrm{BM}(1: 2)$ & $29 \pm 5 \%$ & $11 \pm 3 \%$ & $16 \pm 3 \%$ & $100 \pm 5 \mathrm{~nm}$ & $53 \pm 5 \mathrm{~nm}$ & $72 \pm 7 \mathrm{~nm}$ \\
\hline PCDTBT: PC $_{70} \mathrm{BM}(1: 1)$ & $49 \pm 5 \%$ & $50 \pm 10 \%$ & $50 \pm 10 \%$ & $27 \pm 5 \mathrm{~nm}$ & $24 \pm 5 \mathrm{~nm}$ & $26 \pm 5 \mathrm{~nm}$ \\
\hline PCPDTBT: PC $_{70} B M(1: 1)$ & $53 \pm 5 \%$ & $47 \pm 3 \%$ & $39 \pm 3 \%$ & $63 \pm 5 \mathrm{~nm}$ & $37 \pm 6 \mathrm{~nm}$ & $60 \pm 8 \mathrm{~nm}$ \\
\hline
\end{tabular}

the optical constants and degree of anisotropy that is beyond the original scope of this paper. For completeness and ease of comparison, case examples of $1: 1(\mathrm{w}: \mathrm{w})$ PCDTBT: $\mathrm{PC}_{70} \mathrm{BM}$ and PCPDTBT: $\mathrm{PC}_{70} \mathrm{BM}$ blends are also included in Table 2.

One interesting application of the Raman methodology described in this work consists of forming Raman-based thickness and composition images of polymer:fullerene blends with a lateral resolution of a few hundreds of nanometres. As a first demonstration, we present in Fig. 5(a-c) Raman images of $1: 1$ $(\mathrm{w}: \mathrm{w})$ PCPDTBT: $\mathrm{PC}_{70} \mathrm{BM}$ blends in which the methodology is applied to fit the acquired spectra. The images were recorded centred at a fullerene aggregate, whose thickness non-uniformity creates a visible surrounding halo [Fig. 5(a)]. Fig. 5(b and c) demonstrate how Raman is sensitive enough to accurately quantify the variation in film thickness associated with the halo, and also how the polymer content drops at the aggregate. The composition profile was calibrated according to a corrected curve extracted from

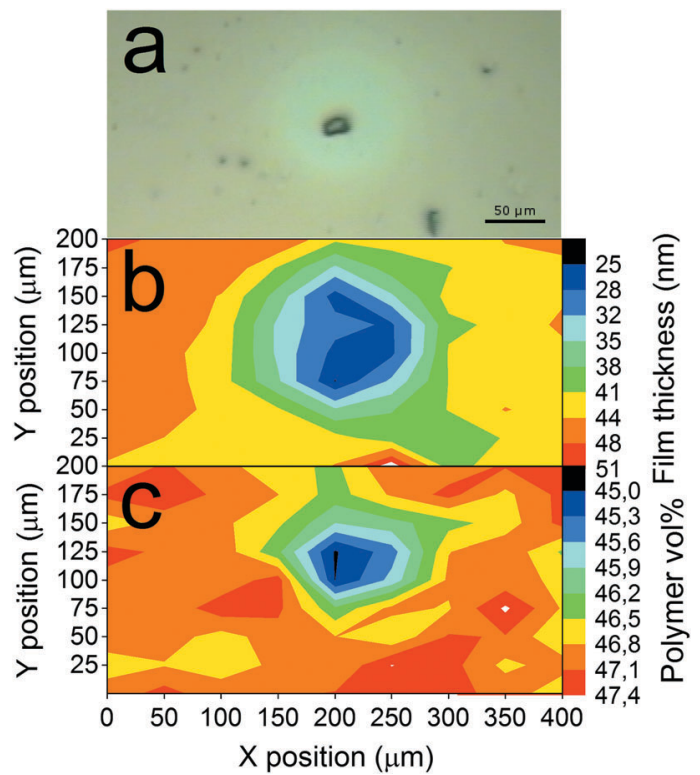

Fig. 5 Raman-based imaging of a 1:1 (w: w) PCPDTBT: PC $_{70}$ BM blended thin film. A total of 81 spectra were taken to build the images, measured at the grid positions defined by the ticks at the axis. (a) Region analysed as seen through the optical microscope; (b) thickness in $\mathrm{nm}$ and (c) polymer volumetric fraction. Note that the area sampled using confocal Raman spectroscopy $\left(400 \times 200 \mu \mathrm{m}^{2}\right)$ is hundreds of times larger than the typical area covered in scanning probe microscopies $\left(20 \times 20 \mu \mathrm{m}^{2}\right)$ and close to the dimensions of the light spot in ellipsometry $\left(200 \times 200 \mu \mathrm{m}^{2}\right)$, thus linking both characterization scales. the data plotted in Fig. 4(a). Raman images suggest that the volumetric distribution of the polymer:fullerene blend is rather homogeneous throughout the sample except at the defects (aggregates).

\section{Conclusions}

We have successfully demonstrated that Raman scattering is a powerful tool for the accurate determination of film thickness and volumetric composition in polymer:fullerene blend thin films used in organic photovoltaics. The applicable thickness range extends from a few monolayers up to several hundreds of nanometres depending on the optical constants of the film and the stack in which it is embedded. To do so, we have developed a methodology which describes the dependence of the Raman intensity as a function of film thickness and composition via the transfer matrix method for the incoming and Ramanscattered light. We predicted and measured an interferencelike pattern of the Raman intensity as a function of film thickness and demonstrated the capabilities of the method to infer film thicknesses below the first interference maximum within an error margin of $c a .10 \mathrm{~nm}$. Regarding composition, the method returns values consistent with ellipsometry $( \pm 5 \mathrm{vol} \%$ in the best scenario, $\pm 10 \mathrm{vol} \%$ otherwise) provided the chemical microenvironment of the different domains of the blend does not deviate too much from the neat films used to estimate reference Raman cross-sections. Hence the Raman methodology described in this work is especially suitable for those polymer:fullerene blends which include amorphous polymers or, alternatively, systems whose Raman spectrum is not very sensitive to variations of the chemical environment (structural changes, degree of order, crystallinity and miscibility). When this is not the case, a calibration curve for composition can be deduced by comparing Raman and ellipsometry.

When exploited in combination with confocal Raman imaging setups, our methodology entails a significant improvement regarding the ease and speed of characterization of thin films compared to scanning probe microscopies. In the latter case the high lateral resolution $(20-30 \mathrm{~nm})$ does not balance the low throughput when dealing with large film areas. The intrinsic diffraction-limited lateral resolution of the confocal Raman setup (ca. $300 \mathrm{~nm}$ ) makes this technique appropriate for characterizing the large scale inhomogeneities that occur during wet deposition processes extending from a few microns up to centimetres. Furthermore, the present methodology can be applied to any 
thin film technology in which multiple Raman-active materials are either blended or stacked as multilayers. Some potential applications include in-line monitoring and quality control during film fabrication and optimization of optoelectronic devices in which film composition and thickness play a crucial role. In this sense, the methodology presented here offers the possibility of measuring film thickness and composition in functional (ready-to-use) devices such as organic solar cells without significant truncation of their operation. This is achieved by properly selecting the laser power to prevent photodegradation from occurring.

\section{Experimental details}

Confocal Raman spectroscopy was performed in two different setups: (1) a LabRam HR800 spectrometer (Jovin Yvon, 600 lines per mm grating) coupled to an Olympus microscope with a $20 \times$ objective $(\mathrm{NA}=0.35)$ in backscattering geometry; and (2) a WITec Alpha300RA (1200 lines per mm grating) with different Zeiss objectives $(10 \times, \mathrm{NA}=0.25 ; 40 \times$ with correction collar, $\mathrm{NA}=0.6 ; 50 \times, \mathrm{NA}=0.8)$. The excitation source corresponds to either the $633 \mathrm{~nm}$ line of a He-Ne laser, a 514/488 $\mathrm{nm} \mathrm{Ar}^{+}$ line or a solid-state $488 \mathrm{~nm}$ laser (WITec). Laser irradiances were kept below $350 \mathrm{~W} \mathrm{~cm}^{-2}$ to avoid photodegradation and laser-induced heating of the films.

All Raman measurements taken with the LabRam HR800 spectrometer were performed at room temperature and under vacuum, whereas those extracted using the WITec Alpha300RA were performed on-the-fly, under ambient conditions and employing low acquisition times $(<500 \mathrm{~ms})$. Vacuum is necessary to avoid oxygen-driven photodegradation of conjugated molecules upon laser exposure for prolonged times ( $>5$ seconds), which has a strong influence on the Raman to photoluminescence background ratio; see Fig. S6-S9 in the ESI. $\dagger$ Spectra for each sample were extracted at a minimum of three different positions in the sample and averaged to account for possible inhomogeneities. The integration time and the number of integrating cycles were adjusted to avoid photodegradation. In all cases the Raman spectra were acquired focusing the laser at the film/substrate interface rather than maximizing the scattered intensity. In the latter case the experimental trends were not properly reproduced by our model. Raw data were fitted using the open-source Fityk software $^{51}$ as well as homemade fitting routines in MATLAB to automatically treat the datasets obtained as Raman maps.

Samples with thickness gradients were fabricated following a method based on accelerated blade coating. ${ }^{52}$ In this deposition technique a controlled volume of ink (with a low solid content ranging from 20 to $40 \mathrm{mg} \mathrm{mL} \mathrm{mL}^{-1}$ ) containing the conjugated molecule(s) is deposited on top of a substrate and then spread over its surface employing a metallic blade or applicator. The velocity of the applicator with respect to the substrate determines the final film thickness achieved. A thickness gradient can be readily obtained accelerating/decelerating the blade during the deposition process. Chlorobenzene was used as solvent in all cases. Glass substrates were previously cleaned with acetone and isopropyl alcohol to assure proper ink wettability and film uniformity. The temperature of the blade coater stage was kept fixed at $60{ }^{\circ} \mathrm{C}$ to ensure controlled evaporation of the solvent and drying kinetics.

The Raman characterization of samples with a thickness gradient was performed using a motorized XY stage along a straight line (LabRam HR800) or an elongated slab formed by at least three parallel lines (Alpha300RA). Alternative thickness measurements were then obtained by profilometry making use of previous localization marks made on the samples.

Variable-angle spectroscopic ellipsometry (VASE) was acquired at several angles of incidence ranging from 55 to 75 degrees using a GES-6E rotating polarizer spectroscopic ellipsometer (SEMILAB) coupled to a charge-coupled device (CCD) detector. The data were analysed using the Winelli 2 piece of software. The blends were modeled using the Bruggeman effective medium approximation for which the corresponding refractive indices of the materials have been published elsewhere. ${ }^{16,53}$

$(6,6)$-Phenyl- $\mathrm{C}_{61}$-butyric acid methyl ester (PCBM) and $(6,6)$ phenyl- $\mathrm{C}_{71}$-butyric acid ester $\left(\mathrm{PC}_{70} \mathrm{BM}\right)$ were obtained from Solenne BV. Indene- $\mathrm{C}_{60}$ bisadduct (ICBA), regioregular poly(3hexylthiophene) (rr-P3HT), poly(2,7-(9,9-di- $n$-octylfluorene-altbenzothiadiazole)) (PFBT), poly(9,9-dioctylfluorene) (PFO) and poly[2-methoxy-5-(2-ethylhexyloxy)-1,4-phenylenevinylene] (MEHPPV) were purchased from Sigma Aldrich. Poly[2,6-(4,4-bis-(2ethylhexyl)- $4 H$-cyclopenta[2,1- $\left.b ; 3,4-b^{\prime}\right]$ dithiophene)-alt-4,7(2,1,3benzothiadiazole)] (PCPDTBT) was purchased from Organic Nano Electronic. Poly[[9-(1-octylnonyl)-9H-carbazole-2,7-diyl]2,5-thiophenediyl-2,1,3-benzothiadiazole-4,7-diyl-2,5-thiophenediyl] (PCDTBT) and poly[(5,6-difluoro-2,1,3-benzothiadiazol-4,7-diyl)-alt$\left(3,3^{\prime \prime \prime}\right.$-di(2-octyldodecyl) $2,2^{\prime} ; 5^{\prime}, 2^{\prime \prime} ; 5^{\prime \prime}, 2^{\prime \prime \prime}$-quaterthiophen-5, $5^{\prime \prime \prime}$-diyl)] (PffBT4T-2OD, also known as PCE11) were purchased from Ossila. All materials were used as received without further purification. Benzotrithiophene-diketopyrrolopyrrole copolymer (BTT-DPP), thieno[3,2- $b]$ thiophene-diketopyrrolopyrrole copolymers (DPPTT-T-1 and DPP-TT-T-3) and indacenodithiophene-benzothidiazole copolymer (C16-IDTBT) were synthetized at Imperial College as reported elsewhere. ${ }^{38,48,54}$

\section{Appendix}

The successful analysis of the Raman spectra of a binary mixture requires using both the film thickness and the volumetric fraction of one of the components as free parameters during the modeling. Since we consider that the optical constants of the blended system vary linearly with film composition $\left(\varepsilon_{\mathrm{mix}}=v_{1} \varepsilon_{1}+v_{2} \varepsilon_{2}\right.$ and $\varepsilon_{\mathrm{mix}}^{R}=$ $\left.v_{1} \varepsilon_{1}^{R}+v_{2} \varepsilon_{2}^{R}\right)$, the shape of the Raman intensity curve as a function of film thickness is modified accordingly. Regarding the modeling of the scattered light, we assume that its wavelength corresponds to that of the most intense Raman band in the spectral region analyzed. In polymer:fullerene blends this approximation usually leads to a constant scattered wavelength such that $\lambda_{\text {mix }}^{R}=\lambda_{\text {polymer }}^{R} \neq f(v)$. This assumption is valid as (i) the high Raman cross-section of conjugated polymers compared to fullerenes implies that their characteristic Raman bands are 


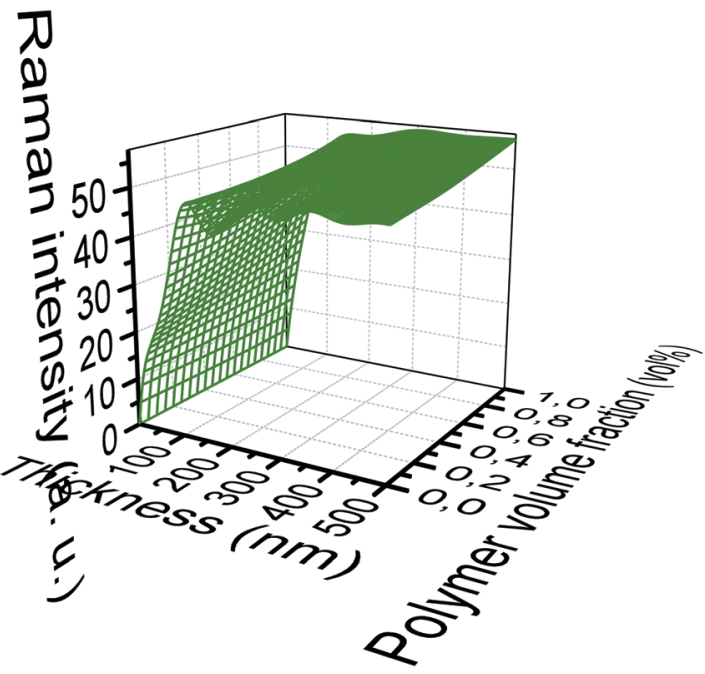

Fig. 6 Three-dimensional surface plot of the modeled Raman intensity as a function of polymer volume content and film thickness for a PCPDTBT:PC 70 BM blend system under $514 \mathrm{~nm}$ excitation.

usually the most intense ones in the majority of composition profiles; and (ii) the spectral window we typically deal with during the fit (1000-1700 $\left.\mathrm{cm}^{-1}\right)$ is small enough to neglect the very small variations that occur in the interference curve as a function of the scattered wavelength.

Fig. 6 illustrates how the amplitude and the film thickness associated with the Raman intensity maxima of the oscillating curve are shifted as a function of the polymer content in a PCPDTBT:PC ${ }_{70} \mathrm{BM}$ blend under $514 \mathrm{~nm}$ excitation, as obtained numerically solving the following integral:

$$
I_{\mathrm{R}, \text { mix }}\left(d, v_{u}\right) \propto \int_{0}^{d}\left|E_{\mathrm{R}}\left(x, v_{u}\right)\right|^{2} \mathrm{~d} x
$$

The general trend in this case indicates that the higher the polymer content, the more damped the intensity oscillations are as a result of the lower refractive index and the higher extinction coefficient compared to the neat fullerene film. When using a new Raman setup, we should obtain at least the effective cross-section of one of the components forming the blend to then estimate the cross-section due to the other material according to the ratios provided in Table 1 for neat films (obtained fitting eqn (4) to experimental data). Finally, the Raman spectra acquired for any blended system formed by these materials can be fitted according to eqn (5) by interpolating the film thickness and composition in the corresponding integral (eqn (A1) and Fig. 6).

\section{Acknowledgements}

We are indebted to Bernhard Dörling from ICMAB for the development of the accelerated blade coating platform. We also acknowledge Antonio Sánchez-Díaz from ICMAB for the evaporation of $\mathrm{MoO}_{3} / \mathrm{Ag}$ electrodes. The Spanish Ministerio de Economía y Competitividad (MINECO) is gratefully acknowledged for its support through Grant No. SEV-2015-0496 in the framework of the Spanish Severo Ochoa Centre of Excellence program and through Grants No. MAT2012-37776, MAT2015-70850-P and CSD2010-00044 (Consolider NANOTHERM). We also acknowledge financial support from the European Research Council through project ERC CoG648901. M. S. V. acknowledges support from the Engineering and Physical Sciences Research Council (EPSRC) via a post-graduate research studentship. J. N. and X. S. thank the EPSRC for support via grants EP/M025020/1 and EP/K029843/1. M. S. V. and X. S. acknowledge additional support from European COST Action MP1307 (StableNextSol) for inter-institutional research visits.

\section{Notes and references}

1 Z. He, C. Zhong, S. Su, M. Xu, H. Wu and Y. Cao, Nat. Photonics, 2012, 6, 593-597.

2 J. You, L. Dou, K. Yoshimura, T. Kato, K. Ohya, T. Moriarty, K. Emery, C.-C. Chen, J. Gao, G. Li and Y. Yang, Nat. Commun., 2013, 4, 1446.

3 J. Nelson, Mater. Today, 2011, 14, 462-470.

4 E. Klimov, W. Li, X. Yang, G. G. Hoffmann and J. Loos, Macromolecules, 2006, 39, 4493-4496.

5 H. Hoppe, M. Niggemann, C. Winder, J. Kraut, R. Hiesgen, A. Hinsch, D. Meissner and N. S. Sariciftci, Adv. Funct. Mater., 2004, 14, 1005-1011.

6 C. R. McNeill, B. Watts, L. Thomsen, H. Ade, N. C. Greenham and P. C. Dastoor, Macromolecules, 2007, 40, 3263-3270.

7 R. C. Masters, A. J. Pearson, T. S. Glen, F.-C. Sasam, L. Li, M. Dapor, A. M. Donald, D. G. Lidzey and C. Rodenburg, Nat. Commun., 2015, 6, 6928.

8 N. Kumar, A. Zoladek-Lemanczyk, A. A. Y. Guilbert, W. Su, S. M. Tuladhar, T. Kirchartz, B. C. Schroeder, I. McCulloch, J. Nelson, D. Roy and F. A. Castro, Nanoscale, 2017, 9, 2723-2731.

9 A. Hammiche, H. M. Pollock, M. Reading, M. Claybourn, P. H. Turner and K. Jewkes, Appl. Spectrosc., 1999, 53, 810-815.

10 F. Huth, A. Govyadinov, S. Amarie, W. Nuansing, F. Keilmann and R. Hillenbrand, Nano Lett., 2012, 12, 3973-3978.

11 F. Lu, M. Jin and M. A. Belkin, Nat. Photonics, 2014, 8, 307-312.

12 I. Burgués-Ceballos, $\mathrm{PhD}$ thesis, Universitat Autònoma de Barcelona, 2014.

13 L. Hou, E. Wang, J. Bergqvist, B. V. Andersson, Z. Wang, C. Müller, M. Campoy-Quiles, M. R. Andersson, F. Zhang and O. Inganäs, Adv. Funct. Mater., 2011, 21, 3169-3175.

14 F. Nickel, C. Sprau, M. F. G. Klein, P. Kapetana, N. Christ, X. Liu, S. Klinkhammer, U. Lemmer and A. Colsmann, Sol. Energy Mater. Sol. Cells, 2012, 104, 18-22.

15 J. Alstrup, M. Jørgensen, A. J. Medford and F. C. Krebs, ACS Appl. Mater. Interfaces, 2010, 2, 2819-2827.

16 M. Campoy-Quiles, M. I. Alonso, D. D. C. Bradley and L. J. Richter, Adv. Funct. Mater., 2014, 24, 2116-2134.

17 M. D. Schaeberle, C. G. Karakatsanis, C. J. Lau and P. J. Treado, Anal. Chem., 1995, 67, 4316-4321.

18 F. Cerdeira, M. Garriga, M. I. Alonso, J. O. Ossó, F. Schreiber, H. Dosch and M. Cardona, J. Raman Spectrosc., 2013, 44, 597-607. 
19 P. Veerender, V. Saxena, A. K. Chauhan, S. P. Koiry, P. Jha, A. Gusain, S. Choudhury, D. K. Aswal and S. K. Gupta, Sol. Energy Mater. Sol. Cells, 2014, 120, 526-535.

20 J. Razzell-Hollis, W. C. Tsoi and J.-S. Kim, J. Mater. Chem. C, 2013, 1, 6235.

21 W. C. Tsoi, D. T. James, J. S. Kim, P. G. Nicholson, C. E. Murphy, D. D. C. Bradley, J. Nelson and J.-S. Kim, J. Am. Chem. Soc., 2011, 133, 9834-9843.

22 H. M. Liem, P. Etchegoin, K. S. Whitehead and D. D. C. Bradley, Adv. Funct. Mater., 2003, 13, 66-72.

23 E. J. J. Martin, N. Bérubé, F. Provencher, M. Côté, C. Silva, S. K. Doorn and J. K. Grey, J. Mater. Chem. C, 2015, 3, 6058-6066.

24 W. C. Tsoi, W. Zhang, J. Razzell Hollis, M. Suh, M. Heeney, I. McCulloch and J.-S. Kim, Appl. Phys. Lett., 2013, 102, 173302.

25 U. Schmidt, S. Hild, W. Ibach and O. Hollricher, Macromol. Symp., 2005, 230, 133-143.

26 J.-S. Kim, P. K. H. Ho, C. E. Murphy and R. H. Friend, Macromolecules, 2004, 37, 2861-2871.

27 K. F. McCarty, Appl. Opt., 1987, 26, 4482.

28 D. Blue, K. Helwig, M. Moskovits and R. Wolkow, J. Chem. Phys., 1990, 92, 4600-4608.

29 J. W. Ager, D. K. Veirs and G. M. Rosenblatt, J. Chem. Phys., 1990, 92, 2067-2076.

30 Y. Y. Wang, Z. H. Ni, Z. X. Shen, H. M. Wang and Y. H. Wu, Appl. Phys. Lett., 2008, 92, 43121.

31 D. Yoon, H. Moon, Y.-W. Son, J. S. Choi, B. H. Park, Y. H. Cha, Y. D. Kim and H. Cheong, Phys. Rev. B: Condens. Matter Mater. Phys., 2009, 80, 125422.

32 S.-L. Li, H. Miyazaki, H. Song, H. Kuramochi, S. Nakaharai and K. Tsukagoshi, ACS Nano, 2012, 6, 7381-7388.

33 R. J. Nemanich, C. C. Tsai and G. A. N. Connell, Phys. Rev. Lett., 1980, 44, 273-276.

34 O. S. Heavens, Optical Properties of Thin Solid Films, Dover Publications, 1991.

35 L. A. A. Pettersson, L. S. Roman and O. Inganäs, J. Appl. Phys., 1999, 86, 487.

36 P. Peumans, A. Yakimov and S. R. Forrest, J. Appl. Phys., 2003, 93, 3693.

37 X. Zhang, H. Bronstein, A. J. Kronemeijer, J. Smith, Y. Kim, R. J. Kline, L. J. Richter, T. D. Anthopoulos, H. Sirringhaus, K. Song, M. Heeney, W. Zhang, I. McCulloch and D. M. DeLongchamp, Nat. Commun., 2013, 4, 2238.

38 W. Zhang, J. Smith, S. E. Watkins, R. Gysel, M. McGehee, A. Salleo, J. Kirkpatrick, S. Ashraf, T. Anthopoulos, M. Heeney and I. McCulloch, J. Am. Chem. Soc., 2010, 132, 11437-11439.

39 H. Bronstein, D. S. Leem, R. Hamilton, P. Woebkenberg, S. King, W. Zhang, R. S. Ashraf, M. Heeney, T. D. Anthopoulos,
J. De Mello and I. McCulloch, Macromolecules, 2011, 44, 6649-6652.

40 I. McCulloch, R. S. Ashraf, L. Biniek, H. Bronstein, C. Combe, J. E. Donaghey, D. I. James, C. B. Nielsen, B. C. Schroeder and W. Zhang, Acc. Chem. Res., 2012, 45, 714-722.

41 J. T. Rogers, K. Schmidt, M. F. Toney, E. J. Kramer and G. C. Bazan, Adv. Mater., 2011, 23, 2284-2288.

42 P. A. Staniec, A. J. Parnell, A. D. F. Dunbar, H. Yi, A. J. Pearson, T. Wang, P. E. Hopkinson, C. Kinane, R. M. Dalgliesh, A. M. Donald, A. J. Ryan, A. Iraqi, R. A. L. Jones and D. G. Lidzey, Adv. Energy Mater., 2011, 1, 499-504.

43 R. B. Aïch, N. Blouin, A. Bouchard and M. Leclerc, Chem. Mater., 2009, 21, 751-757.

44 N. Blouin, A. Michaud, D. Gendron, S. Wakim, E. Blair, R. Neagu-Plesu, M. Belletête, G. Durocher, Y. Tao and M. Leclerc, J. Am. Chem. Soc., 2008, 130, 732-742.

45 T. Agostinelli, S. Lilliu, J. G. Labram, M. Campoy-Quiles, M. Hampton, E. Pires, J. Rawle, O. Bikondoa, D. D. C. Bradley, T. D. Anthopoulos, J. Nelson and J. E. Macdonald, Adv. Funct. Mater., 2011, 21, 1701-1708.

46 M. Campoy-Quiles, C. Müller, M. Garriga, E. Wang, O. Inganäs and M. I. Alonso, Thin Solid Films, 2014, 571, 371-376.

47 A. J. Pearson, T. Wang, A. D. F. Dunbar, H. Yi, D. C. Watters, D. M. Coles, P. A. Staniec, A. Iraqi, R. A. L. Jones and D. G. Lidzey, Adv. Funct. Mater., 2014, 24, 659-667.

48 I. Meager, R. S. Ashraf, S. Mollinger, B. C. Schroeder, H. Bronstein, D. Beatrup, M. S. Vezie, T. Kirchartz, A. Salleo, J. Nelson and I. McCulloch, J. Am. Chem. Soc., 2013, 135, 11537-11540.

49 S. D. Dimitrov, A. A. Bakulin, C. B. Nielsen, B. C. Schroeder, J. Du, H. Bronstein, I. McCulloch, R. H. Friend and J. R. Durrant, J. Am. Chem. Soc., 2012, 134, 18189-18192.

50 Z. Chen, M. J. Lee, R. Shahid Ashraf, Y. Gu, S. Albert-Seifried, M. Meedom Nielsen, B. Schroeder, T. D. Anthopoulos, M. Heeney, I. McCulloch and H. Sirringhaus, Adv. Mater., 2012, 24, 647-652.

51 M. Wojdyr, J. Appl. Crystallogr., 2010, 43, 1126-1128.

52 C. M. Stafford, K. E. Roskov, T. H. Epps and M. J. Fasolka, Rev. Sci. Instrum., 2006, 77, 23908.

53 M. S. Vezie, S. Few, I. Meager, G. Pieridou, B. Dörling, R. S. Ashraf, A. R. Goñi, H. Bronstein, I. McCulloch, S. C. Hayes, M. Campoy-Quiles and J. Nelson, Nat. Mater., 2016, 15, 746-753.

54 S. D. Dimitrov, C. B. Nielsen, S. Shoaee, P. Shakya Tuladhar, J. Du, I. McCulloch and J. R. Durrant, J. Phys. Chem. Lett., 2012, 3, 140-144. 